\title{
Stationary solutions to a nonlocal fourth-order elliptic obstacle problem
}

\author{
Philippe Laurençot ${ }^{1} \cdot$ Christoph Walker $^{2}$
}

Received: 3 March 2020 / Accepted: 12 March 2020 / Published online: 28 March 2020

(c) The Author(s) 2020

\begin{abstract}
Existence of stationary solutions to a nonlocal fourth-order elliptic obstacle problem arising from the modelling of microelectromechanical systems with heterogeneous dielectric properties is shown. The underlying variational structure of the model is exploited to construct these solutions as minimizers of a suitably regularized energy, which allows us to weaken considerably the assumptions on the model used in a previous article.
\end{abstract}

Keywords MEMS · Stationary solution · Minimizer $\cdot$ Bilaplacian · Variational inequality

Mathematics Subject Classification $35 \mathrm{~K} 86 \cdot 49 \mathrm{~J} 40 \cdot 35 \mathrm{~J} 35 \cdot 35 \mathrm{Q} 74$

\section{Introduction}

Idealized electrostatically actuated microelectromechanical systems (MEMS) are made up of an elastic conducting plate which is clamped on its boundary and suspended above a rigid conducting ground plate. Their dynamics results from the competition between mechanical and electrostatic forces in which the elastic plate is deformed by a Coulomb

Partially supported by the CNRS Projet International de Coopération Scientifique PICS07710.

Dedicated to Michel Chipot on the occasion of his 70th birthday.

Christoph Walker

walker@ifam.uni-hannover.de

Philippe Laurençot

laurenco@math.univ-toulouse.fr

1 Institut de Mathématiques de Toulouse, UMR 5219, Université de Toulouse, CNRS, 31062 Toulouse Cedex 9, France

2 Institut für Angewandte Mathematik, Leibniz Universität Hannover, Welfengarten 1, 30167 Hannover, Germany 
force induced by holding the two plates at different electrostatic potentials. When the electrostatic forces dominate the mechanical ones, the elastic plate comes into contact with the ground plate, thereby generating a short circuit and leading to the occurrence of a touchdown singularity in the related mathematical models, see [5, 6, 9, 12, 18, 19] and the references therein. However, covering the ground plate with a thin insulating layer prevents a direct contact of the two plates and, from a mathematical point of view, features a constraint of obstacle-type which hinders the touchdown singularity. Different models have been developed to take into account the influence of the coating layer deposited on the ground plate, most of them relying on the so-called small aspect ratio approximation and describing the state of the MEMS device by the sole deformation of the elastic plate [2, 3, 14, 15, 20]. A more elaborate model is derived in [13, Section 5], in which the state of the device is not only given by the deformation of the elastic plate, but also by the electrostatic potential in the region between the two plates.

To give a more precise account, we restrict ourselves to a two-dimensional setting, neglecting variations in the transverse horizontal direction, so that the geometry of the device under study herein is the following, see Fig. 1. At rest, the cross-section of the elastic plate is $D:=(-L, L), L>0$, and it is clamped at its boundary $(x, z)=( \pm L, 0)$. The fixed ground plate has the same shape $D$ and is located at $z=-H-d$. It is coated with an insulating layer

$$
\Omega_{1}:=D \times(-H-d,-H)
$$

of thickness $d>0$ with a priori non-uniform dielectric permittivity $\sigma_{1}>0$ and cannot be penetrated by the elastic plate. As a consequence, the vertical displacement $u: \bar{D} \rightarrow \mathbb{R}$ of the elastic plate actually ranges in $[-H, \infty)$ and the contact region $\{(x,-H): x \in D, u(x)=-H\}$ between the insulating layer and the elastic plate might be non-empty. We assume also that the free space

$$
\Omega_{2}(u):=\{(x, z) \in D \times \mathbb{R}:-H<z<u(x)\}
$$

between the upper part of the insulating layer and the elastic plate has uniform permittivity $\sigma_{2}>0$, and we denote the electrostatic potential in the device

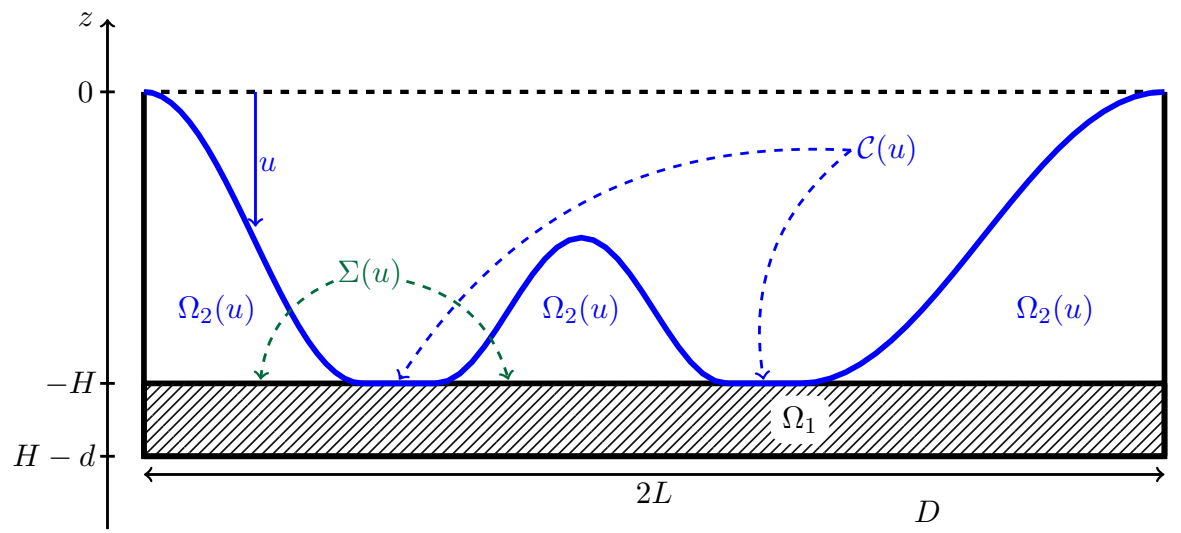

Fig. 1 Geometry of $\Omega(u)$ for a state $u \in \bar{S}_{0}$ with non-empty (and disconnected) coincidence set $\mathcal{C}(u)$ 


$$
\Omega(u):=\{(x, z) \in D \times \mathbb{R}:-H-d<z<u(x)\}=\Omega_{1} \cup \Omega_{2}(u) \cup \Sigma(u)
$$

by $\psi_{u}$, where $\Sigma(u)$ is the interface

$$
\Sigma(u):=\{(x,-H): x \in D, u(x)>-H\} .
$$

According to the model derived in [13, Section 5], equilibrium configurations of the above described device are weak solutions $u \in H_{D}^{2}(D)$ to the fourth-order obstacle problem

$$
\beta \partial_{x}^{4} u-\left(\tau+\alpha\left\|\partial_{x} u\right\|_{L_{2}(D)}^{2}\right) \partial_{x}^{2} u+\partial \mathbb{D}_{\bar{S}_{0}}(u) \ni-g(u) \text { in } D
$$

where

$$
H_{D}^{2}(D):=\left\{v \in H^{2}(D): v( \pm L)=\partial_{x} v( \pm L)=0\right\}
$$

and $\partial \rrbracket_{\bar{S}_{0}}(u)$ denotes the subdifferential in $H_{D}^{2}(D)$ of the indicator function $\rrbracket_{\bar{S}_{0}}$ of the closed convex subset

$$
\bar{S}_{0}:=\left\{v \in H_{D}^{2}(D): v \geq-H \text { in } D\right\}
$$

of $H_{D}^{2}(D)$. We recall that, given $v \in \bar{S}_{0}$, the subdifferential $\partial \rrbracket_{\bar{S}_{0}}(v)$ is the subset of the dual space

$$
H^{-2}(D):=\left(H_{D}^{2}(D)\right)^{\prime}
$$

of $H_{D}^{2}(D)$ given by

$$
\partial \mathbb{\Omega}_{\bar{S}_{0}}(v):=\left\{\xi \in H^{-2}(D):\langle\xi, v-w\rangle_{H_{D}^{2}} \geq 0, w \in \bar{S}_{0}\right\},
$$

where $\langle\cdot, \cdot\rangle_{H_{D}^{2}}$ denotes the duality pairing between $H^{-2}(D)$ and $H_{D}^{2}(D)$. If $v \notin \bar{S}_{0}$, then $\partial \rrbracket_{\bar{S}_{0}}(v):=\emptyset$. While $\partial \rrbracket_{\bar{S}_{0}}(u)$ accounts for the non-penetrability of the insulating layer, the fourth- and second-order terms in (1.1) represent forces due to plate bending and plate stretching, respectively. These forces are balanced by the electrostatic force $g(u)$ acting on the elastic plate, which is derived in [13] and involves the electrostatic potential $\psi_{u}$ in the device. The latter solves the transmission problem

$$
\begin{gathered}
\operatorname{div}\left(\sigma \nabla \psi_{u}\right)=0 \quad \text { in } \Omega(u), \\
\llbracket \psi_{u} \rrbracket=\llbracket \sigma \partial_{z} \psi_{u} \rrbracket=0 \quad \text { on } \Sigma(u), \\
\psi_{u}=h_{u} \quad \text { on } \partial \Omega(u),
\end{gathered}
$$

in the domain $\Omega(u)$, see Fig. 1. In (1.2), $\llbracket \cdot \rrbracket$ denotes the jump across the interface $\Sigma(u)$, the dielectric permittivity $\sigma$ is given by 


$$
\sigma(x, z):= \begin{cases}\sigma_{1}(x, z) & \text { for }(x, z) \in \Omega_{1}, \\ \sigma_{2} & \text { for }(x, z) \in D \times(-H, \infty),\end{cases}
$$

with

$$
\sigma_{1} \in C^{2}\left(\bar{\Omega}_{1}\right), \quad \min _{\bar{\Omega}_{1}} \sigma_{1}>0, \quad \sigma_{2} \in(0, \infty),
$$

and the non-homogeneous Dirichlet boundary conditions $h_{u}$ are given by

$$
h_{u}(x, z):=h(x, z, u(x))=\left\{\begin{array}{l}
h_{1}(x, z, u(x)),(x, z) \in \bar{\Omega}_{1}, \\
h_{2}(x, z, u(x)),(x, z) \in \overline{\Omega_{2}(u)},
\end{array}\right.
$$

where

$$
h_{1}: \bar{D} \times[-H-d,-H] \times[-H, \infty) \rightarrow[0, \infty)
$$

and

$$
h_{2}: \bar{D} \times[-H, \infty) \times[-H, \infty) \rightarrow[0, \infty)
$$

are $C^{2}$-smooth functions satisfying

$$
\begin{gathered}
h_{1}(x,-H, w)=h_{2}(x,-H, w), \quad(x, w) \in D \times[-H, \infty), \\
\sigma_{1}(x,-H) \partial_{z} h_{1}(x,-H, w)=\sigma_{2} \partial_{z} h_{2}(x,-H, w), \quad(x, w) \in D \times[-H, \infty) .
\end{gathered}
$$

We note that $(1.3 \mathrm{~d}-1.3 \mathrm{e})$ imply that $h_{u}$ satisfies

$$
\llbracket h_{u} \rrbracket=\llbracket \sigma \partial_{z} h_{u} \rrbracket=0 \quad \text { on } \Sigma(u)
$$

and thus complies with the transmission conditions (1.2b), see [13, Example 5.5] for an example of functions $h_{1}$ and $h_{2}$ satisfying the above assumptions. With these assumptions, the electrostatic force $g(u)$ is computed in [13, Theorems 1.2 and 1.3]. It has a different expression at contact points between the plates and at points where the elastic plate is strictly above the insulating layer. Specifically, introducing the coincidence set

$$
\mathcal{C}(u):=\{x \in D: u(x)=-H\}
$$

for $u \in \bar{S}_{0}$, the electrostatic force is given by

$$
\begin{aligned}
g(u)(x):= & \mathfrak{g}(u)(x)-\frac{\sigma_{2}}{2}\left[\left(\left(\partial_{x} h_{2}\right)_{u}\right)^{2}+\left(\left(\partial_{z} h_{2}\right)_{u}+\left(\partial_{w} h_{2}\right)_{u}\right)^{2}\right](x, u(x)) \\
& +\left[\sigma_{1}\left(\partial_{w} h_{1}\right)_{u} \partial_{z} \psi_{u, 1}\right](x,-H-d)
\end{aligned}
$$

for $x \in D$, where 


$$
\begin{array}{r}
\mathfrak{g}(u)(x) \\
:= \begin{cases}\frac{\sigma_{2}}{2}\left(1+\left(\partial_{x} u(x)\right)^{2}\right)\left[\partial_{z} \psi_{u, 2}-\left(\partial_{z} h_{2}\right)_{u}-\left(\partial_{w} h_{2}\right)_{u}\right]^{2}(x, u(x)), & x \in D \backslash \mathcal{C}(u), \\
\frac{\sigma_{2}}{2}\left[\frac{\sigma_{1}}{\sigma_{2}} \partial_{z} \psi_{u, 1}-\left(\partial_{z} h_{2}\right)_{u}-\left(\partial_{w} h_{2}\right)_{u}\right]^{2}(x,-H), & x \in \mathcal{C}(u),\end{cases}
\end{array}
$$

and $\left(\psi_{u, 1}, \psi_{u, 2}\right):=\left(\psi_{u} \mathbf{1}_{\bar{\Omega}_{1}}, \psi_{u} \mathbf{1}_{\Omega_{2}(u)}\right)$. It is readily seen from (1.5) that $g(u)$ features a nonlinear and nonlocal dependence on $u$, the latter being due to the terms involving $\psi_{u}$ in (1.5).

The investigation of the solvability of (1.1-1.2) is initiated in [13], exploiting the variational structure underlying the derivation of (1.1-1.2) which implies that solutions to (1.1) are critical points in $\bar{S}_{0}$ of an energy functional $E$, which is actually the total energy of the device. Specifically,

$$
E(u):=E_{m}(u)+E_{e}(u)
$$

consists of the mechanical energy

$$
E_{m}(u):=\frac{\beta}{2}\left\|\partial_{x}^{2} u\right\|_{L_{2}(D)}^{2}+\left(\frac{\tau}{2}+\frac{\alpha}{4}\left\|\partial_{x} u\right\|_{L_{2}(D)}^{2}\right)\left\|\partial_{x} u\right\|_{L_{2}(D)}^{2}
$$

and the electrostatic energy

$$
E_{e}(u):=-\frac{1}{2} \int_{\Omega(u)} \sigma\left|\nabla \psi_{u}\right|^{2} \mathrm{~d}(x, z)
$$

Then (1.1) subject to (1.5) is the Euler-Lagrange equation for minimizers of $E$ in $\bar{S}_{0}$, see [13], and $g(u)$ defined in (1.5) corresponds to the (directional) derivative of $E_{e}(u)$ with respect to $u$, see [13, Theorem 1.4]. The existence of solutions to (1.1) is established in [13, Section 5] by showing that the energy functional has at least one minimizer on $\bar{S}_{0}$. This, however, requires additional assumptions ensuring that the electrostatic energy $E_{e}(u)$ does not grow faster than $\|u\|_{H^{1}(D)}^{2}$ as well as the coercivity of the energy functional $E$. More precisely, to guarantee the former (see (2.2) below) we assume that there are constants $m_{i}>0, i=1,2,3$, such that

$$
\left|\partial_{x} h_{1}(x, z, w)\right|+\left|\partial_{z} h_{1}(x, z, w)\right| \leq \sqrt{m_{1}+m_{2} w^{2}}, \quad\left|\partial_{w} h_{1}(x, z, w)\right| \leq \sqrt{m_{3}},
$$

for $(x, z, w) \in \bar{D} \times[-H-d,-H] \times[-H, \infty)$ and

$$
\left|\partial_{x} h_{2}(x, z, w)\right|+\left|\partial_{z} h_{2}(x, z, w)\right| \leq \sqrt{\frac{m_{1}+m_{2} w^{2}}{H+w}}, \quad\left|\partial_{w} h_{2}(x, z, w)\right| \leq \sqrt{\frac{m_{3}}{H+w}}
$$

for $(x, z, w) \in \bar{D} \times[-H, \infty) \times[-H, \infty)$. The existence result from [13] then reads: 
Proposition 1.1 [13, Theorems 5.1 and 5.3] Let $\beta>0$ and $\tau, \alpha \geq 0$. Assume that (1.3), (1.7), (1.8), and (1.9) hold, and that the ground plate and the elastic plate are kept at constant, but different, electrostatic potentials; that is, there is $V>0$ such that

$$
\begin{gathered}
h_{1}(x,-H-d, w)=0, \quad(x, w) \in \bar{D} \times[-H, \infty), \\
h_{2}(x, w, w)=V, \quad(x, w) \in \bar{D} \times[-H, \infty) .
\end{gathered}
$$

If

$$
\max \{\alpha, \mathfrak{R}\}>0
$$

with

$$
\mathfrak{\Re}:=\beta-4 L^{2}\left[(d+1) \max \left\{\left\|\sigma_{1}\right\|_{L_{\infty}\left(\Omega_{1}\right)}, \sigma_{2}\right\}\left(12 m_{2} L^{2}+2 m_{3}\right)-\tau\right]_{+},
$$

then there is at least one solution $u \in \bar{S}_{0}$ to the variational inequality (1.1) in the following sense: for all $w \in \bar{S}_{0}$,

$$
\begin{aligned}
& \int_{D}\left\{\beta \partial_{x}^{2} u \partial_{x}^{2}(w-u)+\left[\tau+\alpha\left\|\partial_{x} u\right\|_{L_{2}(D)}^{2}\right] \partial_{x} u \partial_{x}(w-u)\right\} \mathrm{d} x \\
& \quad \geq-\int_{D} g(u)(w-u) \mathrm{d} x
\end{aligned}
$$

where $g(u)$ is given by (1.5) and $\psi_{u}$ is the solution to (1.2). Here, we interpret $\partial_{x}^{4} u$ for $u \in \bar{S}_{0}$ as an element of $H^{-2}(D)$ by virtue of

$$
\left\langle\partial_{x}^{4} u, \phi\right\rangle_{H_{D}^{2}}:=\int_{D} \partial_{x}^{2} u \partial_{x}^{2} \phi \mathrm{d} x, \quad \phi \in H_{D}^{2}(D) .
$$

Moreover, this solution can be obtained as a minimizer of $E$ on $\bar{S}_{0}$.

Obviously, a first step towards a full proof of Proposition 1.1 is to solve the transmission problem (1.2) for the electrostatic potential $\psi_{u}$ with sufficient regularity in order to give a meaning to the function $g$ along with deriving suitable continuity properties. We refer to Sect. 2.1 for a detailed account on this issue (see in particular Lemmas 2.1 and 2.2 where these results are recalled). A second essential step in the proof of Proposition 1.1 consists of deriving the coercivity of the energy functional $E$ on $\bar{S}_{0}$. This property is ensured by assumption (1.10) (along with (1.7)). In particular, if $\alpha>0$, then the mechanical energy $E_{m}$ involves a super-quadratic term which allows a compensation of the negative contribution from the electrostatic energy $E_{e}$.

Remark 1.2 Note that (1.8) implies

$$
\partial_{w} h_{1}(x,-H-d, w)=0, \quad(x, w) \in \bar{D} \times[-H, \infty),
$$

while (1.9) implies 


$$
\partial_{x} h_{2}(x, w, w)=0, \quad(x, w) \in \bar{D} \times[-H, \infty),
$$

and

$$
\partial_{z} h_{2}(x, w, w)+\partial_{w} h_{2}(x, w, w)=0, \quad(x, w) \in \bar{D} \times[-H, \infty),
$$

so that the formula (1.5) for $g(u)$ simplifies and becomes $g(u)=\mathfrak{g}(u)$ in (1.5a). In particular, the function $g(u)$ is non-negative.

The aim of the present work is to establish the existence of a solution to (1.1) under considerably weaker assumptions. In particular, we shall get rid of the technical and somewhat artificial assumption (1.10). Since (1.10) is obviously satisfied when $\alpha>0$, we shall treat $\alpha$ as zero in the following computations. Moreover, we no longer need a sign for the function $g(u)$ and can slightly weaken assumption (1.9). Indeed, we only require that

$$
\partial_{w} h_{1}(x,-H-d, w)=0, \quad(x, w) \in \bar{D} \times[-H, \infty),
$$

and that there is a number $K>0$ such that

$$
\left|\partial_{x} h_{2}(x, w, w)\right|+\left|\partial_{z} h_{2}(x, w, w)+\partial_{w} h_{2}(x, w, w)\right| \leq K,
$$

for $(x, w) \in \bar{D} \times[-H, \infty)$. Clearly, (1.8-1.9) imply (1.11). Also note that, due to (1.11a), the last term in the definition of $g(u)$ in $(1.5 \mathrm{a})$ vanishes, i.e. $g(u)$ reduces to

$$
g(u)(x)=\mathfrak{g}(u)(x)-\frac{\sigma_{2}}{2}\left[\left(\left(\partial_{x} h_{2}\right)_{u}\right)^{2}+\left(\left(\partial_{z} h_{2}\right)_{u}+\left(\partial_{w} h_{2}\right)_{u}\right)^{2}\right](x, u(x)) .
$$

With these assumptions we can now formulate the main result of the present paper.

Theorem 1.3 Let $\beta>0, \tau \geq 0$, and $\alpha=0$. Assume that (1.3), (1.7), and (1.11) hold. Then there is at least one solution $u \in \bar{S}_{0}$ to the variational inequality (1.1) in the sense of Proposition 1.1. More precisely, the functional $E$ is bounded from below on $\bar{S}_{0}$ and has a minimizer on $\bar{S}_{0}$ which is a weak solution to (1.1).

Since we no longer impose assumption (1.10) in Theorem 1.3, the boundedness from below of the functional $E$ is a priori unclear, due to the negative contribution from the electrostatic energy $E_{e}$. We thus shall work with regularized coercive functionals instead (see (2.1) below) and use comparison principle arguments to derive a priori bounds on minimizers of the regularized functionals, see Sect. 2 below. We shall then prove that cluster points of these minimizers are actually minimizers of the original functional $E$. The full proof of Theorem 1.3 is given in Sect. 2 and relies on an idea introduced previously in a related work [10].

Remark 1.4 Theorem 1.3 remains valid when $\alpha>0$ and then only requires the assumptions (1.3) and (1.7). Indeed, the existence of a minimizer of $E$ on $\bar{S}_{0}$ is shown as in the proof of [13, Theorem 5.1] and this minimizer is a weak solution to (1.1) as a consequence of [13, Theorem 5.3]. 
Finally, we provide an additional property of weak solutions to (1.1) when the potentials applied on the elastic plate and the ground plate are constant.

Corollary 1.5 Suppose (1.3), (1.8), and (1.9). If the coincidence set $\mathcal{C}(u) \subset D$ of a solution $u \in \bar{S}_{0}$ to (1.1) is non-empty, then it is an interval.

\section{Proof of Theorem 1.3 and Corollary 1.5}

\subsection{Auxiliary results}

Let us emphasize that the function $g(u)$ defined in (1.5) involves gradient traces of the electrostatic potential $\psi_{u}$, the latter solving the transmission problem (1.2) posed on the non-smooth domain $\Omega(u)$ which possesses corners. In addition, $\Omega_{2}(u)$ need not be connected, but may consist of several components with non-Lipschitz boundaries (see Fig. 1), so that traces have first to be given a meaning. While the existence of a unique variational solution $\psi_{u} \in h_{u}+H_{0}^{1}(\Omega(u)$ ) to (1.2) readily follows from the Lax-Milgram theorem, the required further regularity for $\psi_{u}$ in order to make sense of its gradient traces is thus far from being obvious. Moreover, $\psi_{u}$ (and hence $g(u)$ ) depends non-locally on $u$ so that continuity properties with respect to the plate deformation $u$ are non-trivial.

Nevertheless, relying on shape optimization methods and Gamma convergence techniques the following result regarding the existence of a solution to the transmission problem (1.2) and its regularity is shown in [13].

Lemma 2.1 Suppose (1.3). For each $u \in \bar{S}_{0}$, there is a unique variational solution $\psi_{u} \in h_{u}+H_{0}^{1}(\Omega(u))$ to (1.2). Moreover,

$$
\psi_{u, 1}=\psi_{u} \mathbf{1}_{\bar{\Omega}_{1}} \in H^{2}\left(\Omega_{1}\right), \quad \psi_{u, 2}=\psi_{u} \mathbf{1}_{\Omega_{2}(u)} \in H^{2}\left(\Omega_{2}(u)\right),
$$

and $\psi_{u}$ is a strong solution to the transmission problem (1.2) satisfying $\sigma \partial_{z} \psi_{u} \in H^{1}(\Omega(u))$. Also,

$$
\inf _{\partial \Omega(u)} h_{u} \leq \psi_{u}(x, z) \leq \sup _{\partial \Omega(u)} h_{u}, \quad(x, z) \in \overline{\Omega(u)} .
$$

Proof The existence, uniqueness, and regularity of the variational solution $\psi_{u}$ to (1.2) follows from [13, Theorem 1.1], while the upper and lower bounds for $\psi_{u}$ are consequences of the weak maximum principle [16, Chapter 7, Exercice 2.2], since $\sigma \in L_{\infty}(\Omega(u))$.

The regularity of $\psi_{u}$ provided by Lemma 2.1 in particular guarantees that $g(u)$ defined in (1.5) is meaningful for $u \in \bar{S}_{0}$. As for the continuity of $g(u)$ with respect to $u \in \bar{S}_{0}$ we recall: 


\section{Lemma 2.2 Suppose (1.3).}

(a) The mapping $g: \bar{S}_{0} \rightarrow L_{2}(D)$ is well-defined, continuous, and bounded on bounded sets, the set $\bar{S}_{0}$ being endowed with the topology of $H^{2}(D)$.

(b) Let $\left(u_{j}\right)_{j \geq 1}$ be a sequence of functions in $\bar{S}_{0}$ such that $u_{j} \rightarrow u$ in $H^{2}(D)$ for some $u \in \bar{S}_{0}$. Then

$$
\lim _{j \rightarrow \infty}\left\|g\left(u_{j}\right)-g(u)\right\|_{L_{2}(D)}=0 \quad \text { and } \quad \lim _{j \rightarrow \infty} E_{e}\left(u_{j}\right)=E_{e}(u) .
$$

Proof Part (a) follows from [13, Theorem 1.4], [13, Corollary 3.14 \& Lemma 3.16], and the continuity of the trace operator from $H^{1}\left(\Omega_{1}\right)$ to $L_{p}(D \times\{-H\})$ for all $p \in[1, \infty)$.

Part (b) follows from [13, Proposition 3.17 \& Corollary 3.12].

\subsection{Minimizers for a regularized energy}

In the following we let $\beta>0$ and $\tau \geq 0$ and assume throughout that (1.3), (1.7), and (1.11) hold. We put

$$
\bar{\sigma}:=\max \left\{\left\|\sigma_{1}\right\|_{L_{\infty}\left(\Omega_{1}\right)}, \sigma_{2}\right\} .
$$

In order to prove Theorem 1.3 it suffices to find a minimizer of the energy functional $E$ on $\bar{S}_{0}$ since any such minimizer satisfies (1.1) according to [13, Theorem 5.3] (note that [13, Theorem 5.3] obviously remains true without imposing [13, Assumption (5.2a)]). However, as mentioned previously, the coercivity of the energy functional $E$ is a priori unclear when dropping assumption (1.10). For this reason we introduce for $k \geq 0$ the regularized functional

$$
\mathcal{E}_{k}(u):=E(u)+\frac{A}{2}\left\|(u-k)_{+}\right\|_{L_{2}(D)}^{2}, \quad u \in \bar{S}_{0},
$$

where $E(u)$ is defined in (1.6) and the constant $A$ given by

$$
A:=8(d+1) \bar{\sigma}\left(\frac{3 m_{2}}{2}+\frac{m_{3}^{2}(d+1) \bar{\sigma}}{\beta}\right)
$$

with constants $m_{j}$ introduced in (1.7). We shall now prove, for each $k>0$, the existence of a minimizer $u_{k}$ of $\mathcal{E}_{k}$ on $\bar{S}_{0}$ and subsequently derive an a priori bound on such minimizers, so that the additional regularizing term drops out in $\mathcal{E}_{k}$. We first show the coercivity of the functional $\mathcal{E}_{k}$.

Lemma 2.3 Given $k \geq H$, there is a constant $c(k)>0$ such that

$$
\mathcal{E}_{k}(u) \geq \frac{\beta}{4}\left\|\partial_{x}^{2} u\right\|_{L_{2}(D)}^{2}+\frac{A}{4}\left\|(u-k)_{+}\right\|_{L_{2}(D)}^{2}-c(k), \quad u \in \bar{S}_{0} .
$$


Proof Let $u \in \bar{S}_{0}$. The variational characterization of $\psi_{u}$, see [13, Lemma 3.2], readily gives

$$
-E_{e}(u)=\frac{1}{2} \int_{\Omega(u)} \sigma\left|\nabla \psi_{u}\right|^{2} \mathrm{~d}(x, z) \leq \frac{1}{2} \int_{\Omega(u)} \sigma\left|\nabla h_{u}\right|^{2} \mathrm{~d}(x, z) .
$$

Thus, invoking (1.3), Young's inequality, and the definition of $\Omega(u)$ we derive

$$
\begin{aligned}
-E_{e}(u) \leq & \int_{\Omega(u)} \sigma\left[\left(\partial_{x} h(x, z, u(x))^{2}+\left(\partial_{w} h(x, z, u(x))^{2}\left(\partial_{x} u\right)^{2}\right] \mathrm{d}(x, z)\right.\right. \\
& +\frac{1}{2} \int_{\Omega(u)} \sigma\left(\partial_{z} h(x, z, u(x))^{2} \mathrm{~d}(x, z)\right. \\
\leq & (d+1) \bar{\sigma}\left\{\frac{3}{2} m_{1}|D|+\frac{3}{2} m_{2}\|u\|_{L_{2}(D)}^{2}+m_{3}\left\|\partial_{x} u\right\|_{L_{2}(D)}^{2}\right\} .
\end{aligned}
$$

Next, since $u \in \bar{S}_{0} \subset H_{D}^{2}(D)$ implies

$$
\int_{D}\left|\partial_{x} u\right|^{2} \mathrm{~d} x=-\int_{D} u \partial_{x}^{2} u \mathrm{~d} x \leq\|u\|_{L_{2}(D)}\left\|\partial_{x}^{2} u\right\|_{L_{2}(D)},
$$

we deduce from Young's inequality

$$
\begin{aligned}
-E_{e}(u) \leq(d+1) \bar{\sigma} & \left\{\frac{3}{2} m_{1}|D|+\frac{3}{2} m_{2}\|u\|_{L_{2}(D)}^{2}+m_{3}\|u\|_{L_{2}(D)}\left\|\partial_{x}^{2} u\right\|_{L_{2}(D)}\right\} \\
\leq(d+1) \bar{\sigma} & \left\{\frac{3}{2} m_{1}|D|+\left(\frac{3 m_{2}}{2}+\frac{m_{3}^{2}(d+1) \bar{\sigma}}{\beta}\right)\|u\|_{L_{2}(D)}^{2}\right\} \\
+ & \frac{\beta}{4}\left\|\partial_{x}^{2} u\right\|_{L_{2}(D)}^{2} .
\end{aligned}
$$

Finally, note that

$$
\begin{aligned}
\|u\|_{L_{2}(D)}^{2} & =\int_{D} u^{2} \mathbf{1}_{[-H, k]}(u) \mathrm{d} x+\int_{D} u^{2} \mathbf{1}_{(k, \infty)}(u) \mathrm{d} x \\
& \leq k^{2} \int_{D} \mathbf{1}_{[-H, k]}(u) \mathrm{d} x+2 \int_{D}(u-k)^{2} \mathbf{1}_{(k, \infty)}(u) \mathrm{d} x+2 k^{2} \int_{D} \mathbf{1}_{(k, \infty)}(u) \mathrm{d} x \\
& \leq 2 k^{2}|D|+2\left\|(u-k)_{+}\right\|_{L_{2}(D)}^{2} .
\end{aligned}
$$

Hence, taking the previous two inequalities into account, the definition of $\mathcal{E}_{k}(u)$ entails

$$
\begin{aligned}
\mathcal{E}_{k}(u) \geq & \frac{\beta}{4}\left\|\partial_{x}^{2} u\right\|_{L_{2}(D)}^{2}+\frac{A}{2}\left\|(u-k)_{+}\right\|_{L_{2}(D)}^{2}-\frac{3}{2}(d+1) \bar{\sigma} m_{1}|D| \\
& \quad-\frac{A}{8}\left(2\left\|(u-k)_{+}\right\|_{L_{2}(D)}^{2}+2 k^{2}|D|\right) \\
\geq & \frac{\beta}{4}\left\|\partial_{x}^{2} u\right\|_{L_{2}(D)}^{2}+\frac{A}{4}\left\|(u-k)_{+}\right\|_{L_{2}(D)}^{2}-c(k)
\end{aligned}
$$

as claimed. 
The just established coercivity now easily yields the existence of a minimizer of $\mathcal{E}_{k}$ on $\bar{S}_{0}$.

Proposition 2.4 For each $k \geq H$, the functional $\mathcal{E}_{k}$ has at least one minimizer $u_{k} \in \bar{S}_{0}$ on $\bar{S}_{0}$; that is,

$$
\mathcal{E}_{k}\left(u_{k}\right)=\min _{\bar{S}_{0}} \mathcal{E}_{k}
$$

Moreover, $u_{k} \in \bar{S}_{0}$ is a weak solution to the variational inequality

$$
\beta \partial_{x}^{4} u_{k}-\tau \partial_{x}^{2} u_{k}+A\left(u_{k}-k\right)_{+}+\partial \rrbracket_{\bar{S}_{0}}\left(u_{k}\right) \ni-g\left(u_{k}\right) \text { in } D .
$$

Proof Clearly, $E_{m}$ defined in (1.6b) is weakly lower semicontinuous on $H^{2}(D)$ while $E_{e}$ is continuous with respect to the weak topology of $H^{2}(D)$ due to Lemma 2.2. Thanks to Lemma 2.3, the direct method of the calculus of variations now easily yields the existence of a minimizer $u_{k} \in \bar{S}_{0}$ of $\mathcal{E}_{k}$ on $\bar{S}_{0}$. In particular,

$$
0 \leq \liminf _{s \rightarrow 0^{+}} \frac{1}{s}\left(\mathcal{E}_{k}\left(u_{k}+s\left(w-u_{k}\right)\right)-\mathcal{E}_{k}\left(u_{k}\right)\right)
$$

for any fixed

$$
w \in S_{0}:=\left\{v \in H_{D}^{2}(D): v>-H \text { in } D\right\} \subset \bar{S}_{0} .
$$

It was shown in [13, Theorem 1.4] that (since $u_{k}+s\left(w-u_{k}\right) \in S_{0}$ for $\left.s \in(0,1)\right)$

$$
\lim _{s \rightarrow 0^{+}} \frac{1}{s}\left(E_{e}\left(u_{k}+s\left(w-u_{k}\right)\right)-E_{e}\left(u_{k}\right)\right)=\int_{D} g\left(u_{k}\right)\left(w-u_{k}\right) \mathrm{d} x .
$$

From the definition of $\mathcal{E}_{k}$ we then obtain by gathering the two limits that

$$
\begin{aligned}
& \int_{D}\left\{\beta \partial_{x}^{2} u_{k} \partial_{x}^{2}\left(w-u_{k}\right)+\tau \partial_{x} u_{k} \partial_{x}\left(w-u_{k}\right)+A\left(u_{k}-k\right)_{+}\left(w-u_{k}\right)\right\} \mathrm{d} x \\
& \quad \geq-\int_{D} g\left(u_{k}\right)\left(w-u_{k}\right) \mathrm{d} x
\end{aligned}
$$

for all $w \in S_{0}$. Since $S_{0}$ is dense in $\bar{S}_{0}$, this inequality also holds for any $w \in \bar{S}_{0}$, and we thus have shown that $u_{k}$ satisfies the variational formulation of (2.4).

\subsection{A priori bounds}

We shall now show that $u_{k}$ is a priori bounded for $k$ large enough (making the additional term in $\mathcal{E}_{k}$ superfluous). To this aim we need an a priori bound for the solution to the fourth-order boundary value problem (2.5) subject to suitable Dirichlet boundary conditions as stated below. The bound relies on the maximum principle for the fourth order operator $\beta \partial_{x}^{4}-\tau \partial_{x}^{2}$ with clamped boundary conditions $[4,8,11$, $17]$. 
Lemma 2.5 Let $G_{0} \geq 0$ and recall that $\beta>0$ and $\tau \geq 0$. For an interval $I:=(a, b) \subset(-L, L)$, let $S_{I} \in C^{4}([a, b])$ denote the unique solution to the boundaryvalue problem

$$
\beta S_{I}^{\prime \prime \prime \prime}-\tau S_{I}^{\prime \prime}=G_{0}, \quad x \in(a, b),
$$

supplemented with one of the boundary conditions:

$$
\begin{gathered}
S_{I}(a)+H=S_{I}^{\prime}(a)=S_{I}(b)+H=S_{I}^{\prime}(b)=0 \quad \text { if } \quad-L<a<b<L, \\
S_{I}(-L)=S_{I}^{\prime}(-L)=S_{I}(b)+H=S_{I}^{\prime}(b)=0 \quad \text { if } \quad-L=a<b<L, \\
S_{I}(a)+H=S_{I}^{\prime}(a)=S_{I}(L)=S_{I}^{\prime}(L)=0 \quad \text { if } \quad-L<a<b=L, \\
S_{I}(-L)=S_{I}^{\prime}(-L)=S_{I}(L)=S_{I}^{\prime}(L)=0 \quad \text { if } \quad-L=a<b=L .
\end{gathered}
$$

Then, there is $\kappa_{0}>0$ depending only on $G_{0}, \beta, L, H$, and $\tau$ (but not on $I=(a, b)$ ) such that

$$
\left|S_{I}(x)\right| \leq \kappa_{0}, \quad x \in[a, b] .
$$

Proof This result has already been observed in [10, Lemma A.1] and we include its proof here only for the sake of completeness. Note that (2.5) subject to one of the boundary conditions (2.6-2.9) indeed admits a unique solution $S_{I}$.

Case 1: $-L<a<b<L$. Set $P(y):=S_{I}(a+(b-a) y)+H$ for $y \in[0,1]$ and note that $P$ solves the boundary-value problem

$$
\begin{aligned}
& \beta P^{\prime \prime \prime \prime}-\tau(b-a)^{2} P^{\prime \prime}=(b-a)^{4} G_{0}, \quad y \in(0,1), \\
& P(0)=P^{\prime}(0)=P(1)=P^{\prime}(1)=0 .
\end{aligned}
$$

Since $G_{0} \geq 0$ we deduce that $P \geq 0$ in $(0,1)$ from a version of Boggio's comparison principle $[4,8,11,17]$. Testing $(2.10)$ by $P$ we get

$$
\beta\left\|P^{\prime \prime}\right\|_{L_{2}(0,1)}^{2}+\tau(b-a)^{2}\left\|P^{\prime}\right\|_{L_{2}(0,1)}^{2}=(b-a)^{4} G_{0} \int_{0}^{1} P(y) \mathrm{d} y .
$$

Since

$$
|P(y)|=\left|\int_{0}^{y}\left(y-y_{*}\right) P^{\prime \prime}\left(y_{*}\right) \mathrm{d} y_{*}\right| \leq\left\|P^{\prime \prime}\right\|_{L_{2}(0,1)},
$$

we infer from the above inequalities that

$$
\beta\|P\|_{L_{\infty}(0,1)}^{2} \leq \beta\left\|P^{\prime \prime}\right\|_{L_{2}(0,1)}^{2} \leq(b-a)^{4} G_{0}\|P\|_{L_{\infty}(0,1)} \leq 16 L^{4} G_{0}\|P\|_{L_{\infty}(0,1)} .
$$

Consequently, $0 \leq P \leq 16 L^{4} G_{0} / \beta$ in $[0,1]$, hence $-H \leq S_{I} \leq 16 L^{4} G_{0} / \beta-H$ in $[a, b]$. 
Case 2: $-L=a<b<L$. Define $Q(y):=y^{2}\left(y^{2}+2(H-1) y+1-3 H\right)$ for $y \in[0,1]$ and note that $Q(0)=Q^{\prime}(0)=Q(1)+H=Q^{\prime}(1)=0$. Set $P(y):=S_{I}(-L+$ $(b+L) y)-Q(y)$ for $y \in[0,1]$. Then, due to (2.5) and (2.7), $P$ solves the boundaryvalue problem

$$
\begin{aligned}
& \beta P^{\prime \prime \prime \prime}-\tau(b+L)^{2} P^{\prime \prime}=(b+L)^{4} G_{0}-\beta Q^{\prime \prime \prime \prime}+\tau(b+L)^{2} Q^{\prime \prime}, \quad y \in(0,1), \\
& P(0)=P^{\prime}(0)=P(1)=P^{\prime}(1)=0 .
\end{aligned}
$$

The arguments of Case 1 give

$$
\begin{aligned}
\beta \| & P \|_{L_{\infty}(0,1)}^{2} \\
& \leq \beta\left\|P^{\prime \prime}\right\|_{L_{2}(0,1)}^{2} \\
& \leq\left[(b+L)^{4} G_{0}+\beta\left\|Q^{\prime \prime \prime \prime}\right\|_{L_{\infty}(0,1)}+\tau(b+L)^{2}\left\|Q^{\prime \prime}\right\|_{L_{\infty}(0,1)}\right]\|P\|_{L_{\infty}(0,1)} \\
& \leq\left[(b+L)^{4} G_{0}+24 \beta+14 \tau(H+1)(b+L)^{2}\right]\|P\|_{L_{\infty}(0,1)} \\
& \leq\left[16 L^{4} G_{0}+24 \beta+56 \tau(H+1) L^{2}\right]\|P\|_{L_{\infty}(0,1)}
\end{aligned}
$$

since $Q^{\prime \prime \prime \prime}=24,\left|Q^{\prime \prime}\right| \leq 14(H+1)$, and $b<L$. Therefore,

$$
\begin{aligned}
\left\|S_{I}\right\|_{L_{\infty}(-L, b)} & \leq\|P\|_{L_{\infty}(0,1)}+\|Q\|_{L_{\infty}(0,1)} \\
& \leq \frac{16 L^{4} G_{0}+24 \beta+56 \tau(H+1) L^{2}}{\beta}+\|Q\|_{L_{\infty}(0,1)} .
\end{aligned}
$$

Case 3: $-L<a<b=L$. Define $P(y):=S_{I}(a+y(L-a))-Q(1-y)$ for $y \in[0,1]$, where $Q$ is as in Case 2. Arguing as in the previous case we obtain the same bound for $\left\|S_{I}\right\|_{L_{\infty}(a, L)}$

Case 4: $-L=a<b=L$. Define $P(y):=S_{I}(-L+2 L y)$ for $y \in[0,1]$. Then, by (2.5) and (2.9), $P$ solves the boundary-value problem

$$
\begin{aligned}
& \beta P^{\prime \prime \prime \prime}-4 \tau L^{2} P^{\prime \prime}=16 L^{4} G_{0}, \quad y \in(0,1), \\
& P(0)=P^{\prime}(0)=P(1)=P^{\prime}(1)=0 .
\end{aligned}
$$

As in Case 1 we deduce that $0 \leq S_{I} \leq 16 L^{4} G_{0} / \beta$ in $[-L, L]$.

The previous lemma now implies the desired a priori bounds on the minimizers $u_{k}$.

Proposition 2.6 There is $\kappa_{0} \geq H>0$ depending only on $K$ introduced in $(1.11 b)$ such that, for all $k \geq H$, the minimizer $u_{k} \in \bar{S}_{0}$ of $\mathcal{E}_{k}$ on $\bar{S}_{0}$ constructed in Proposition 2.4 satisfies $\left\|u_{k}\right\|_{L_{\infty}(D)} \leq \kappa_{0}$.

Proof Let $k \geq H$. We first note that, since $\mathfrak{g}\left(u_{k}\right) \geq 0$ in $D$ by (1.5b), it easily follows from (1.11b) and (1.12) that

$$
g\left(u_{k}\right)(x) \geq-G_{0}:=-\sigma_{2} K^{2}, \quad x \in D
$$


Next, since $u_{k} \in C(\bar{D})$ with $u_{k}( \pm L)=0$, the set $\left\{x \in D: u_{k}(x)>-H\right\}$ is a nonempty open subset of $D$. Owing to [1, IX.Proposition 1.8] we can thus write it as a countable union of open intervals $\left(I_{j}\right)_{j \in J}$. Consider a fixed $j \in J$ and let $S_{I_{j}}$ denote the solution to (2.5) in $I_{j}$ subject to the associated boundary conditions on $\partial I_{j}$ listed in (2.6-2.9), which vary according to whether $\bar{I}_{j} \subset D$ or not. Then Lemma 2.5 yields a constant $\kappa_{0}>H$ (independent of $j \in J$ ) such that

$$
\left\|S_{I_{j}}\right\|_{L_{\infty}\left(I_{j}\right)} \leq \kappa_{0} .
$$

Note that, by definition of $I_{j}$, the function $u_{k}$ restricted to $I_{j}$ satisfies the same boundary conditions on $\partial I_{j}$ as $S_{I_{j}}$. Hence, for $z:=u_{k}-S_{I_{j}} \in H^{2}\left(I_{j}\right)$ we have $z=\partial_{x} z=0$ on $\partial I_{j}$. Moreover, if $\theta \in \mathcal{D}\left(I_{j}\right)$, then $u_{k} \pm \delta \theta \in \bar{S}_{0}$ for $\delta>0$ small enough since $u_{k}>-H$ in the support of $\theta$. Invoking the weak formulation of (2.4) we derive

$$
\pm \delta \int_{I_{j}}\left\{\beta \partial_{x}^{2} u_{k} \partial_{x}^{2} \theta+\tau \partial_{x} u_{k} \partial_{x} \theta+A\left(u_{k}-k\right)_{+} \theta\right\} \mathrm{d} x \geq \mp \delta \int_{I_{j}} g\left(u_{k}\right) \theta \mathrm{d} x
$$

hence

$$
\int_{I_{j}}\left\{\beta \partial_{x}^{2} u_{k} \partial_{x}^{2} \theta+\tau \partial_{x} u_{k} \partial_{x} \theta+A\left(u_{k}-k\right)_{+} \theta\right\} \mathrm{d} x=-\int_{I_{j}} g\left(u_{k}\right) \theta \mathrm{d} x
$$

Thus, we conclude that $z=u_{k}-S_{I_{j}} \in H^{2}\left(I_{j}\right)$ weakly solves the boundary value problem

$$
\begin{gathered}
\beta \partial_{x}^{4} z-\tau \partial_{x}^{2} z=-G_{0}-g\left(u_{k}\right)-A\left(u_{k}-k\right)_{+} \text {in } I_{j}, \\
z=\partial_{x} z=0 \text { on } \partial I_{j} .
\end{gathered}
$$

Now, since $g\left(u_{k}\right)+A\left(u_{k}-k\right)_{+} \in L_{2}\left(I_{j}\right)$ by Lemma 2.2 (a), classical elliptic regularity theory [7] entails that $z=u_{k}-S_{I_{j}} \in H^{4}\left(I_{j}\right)$ is a strong solution to (2.14). Furthermore, it follows from (2.12) and the non-negativity of $A\left(u_{k}-k\right)_{+}$that the right-hand side of (2.14a) is a non-positive function. Boggio's comparison principle [4, 8, 11, 17] then implies that $z=u_{k}-S_{I_{j}}<0$ in $I_{j}$. Consequently, we infer from (2.13) that $\left\|u_{k}\right\|_{L_{\infty}\left(I_{j}\right)} \leq \kappa_{0}$. Since $\kappa_{0} \quad$ is independent of $j \in J$ and $\left(I_{j}\right)_{j \in J}$ covers $\left\{x \in D: u_{k}(x)>-H\right\}$, the assertion follows.

\subsection{Proof of Theorem 1.3}

We are now in a position to finish off the proof of Theorem 1.3. Indeed, if $u_{k} \in \bar{S}_{0}$ is the minimizer of the functional $\mathcal{E}_{k}$ on $\bar{S}_{0}$ provided by Proposition 2.4 , then $-H \leq u_{k} \leq \kappa_{0}$ in $D$ due to Proposition 2.6. Consequently, for $k \geq \kappa_{0}$ we have

$$
E\left(u_{k}\right)=\mathcal{E}_{\kappa_{0}}\left(u_{k}\right)=\mathcal{E}_{k}\left(u_{k}\right) \leq \mathcal{E}_{k}(v)=E(v)+\frac{A}{2}\left\|(v-k)_{+}\right\|_{L_{2}(D)}^{2}, \quad v \in \bar{S}_{0} .
$$


Now, since $0 \in \bar{S}_{0}$ it follows from Lemma 2.3 that, for $k \geq \kappa_{0}$,

$$
\frac{\beta}{4}\left\|\partial_{x}^{2} u_{k}\right\|_{L_{2}(D)}^{2} \leq \mathcal{E}_{\kappa_{0}}\left(u_{k}\right)+c\left(\kappa_{0}\right) \leq \mathcal{E}_{\kappa_{0}}(0)+c\left(\kappa_{0}\right)=E(0)+c\left(\kappa_{0}\right) .
$$

Thus, $\left(u_{k}\right)_{k \geq \kappa_{0}}$ is bounded in $H^{2}(D)$ so that there is a subsequence (not relabeled) converging weakly in $H^{2}(D)$ and strongly in $H^{1}(D)$ towards some $u_{*} \in \bar{S}_{0}$. Since $E_{m}$ is weakly lower semicontinuous on $H^{2}(D)$ and since $E_{e}$ is continuous with respect to the weak topology of $H^{2}(D)$ owing to Lemma 2.2 (b), we obtain from (2.15) that

$$
E\left(u_{*}\right) \leq E(v), \quad v \in \bar{S}_{0},
$$

recalling that the continuous embedding of $H^{1}(D)$ in $C(\bar{D})$ readily implies that

$$
\lim _{k \rightarrow \infty}\left\|(v-k)_{+}\right\|_{L_{2}(D)}=0, \quad v \in \bar{S}_{0} .
$$

Therefore, $u_{*} \in \bar{S}_{0}$ minimizes $E$ on $\bar{S}_{0}$. Now [13, Theorem 5.3] entails that $u_{*}$ satisfies the variational inequality (1.1). Alternatively, one can use the weak convergence in $H^{2}(D)$ and the strong convergence in $H^{1}(D)$ of $\left(u_{k}\right)_{k \geq \kappa_{0}}$ to $u_{*}$ to pass to the limit $k \rightarrow \infty$ in (2.4), observing that $\left(g\left(u_{k}\right)\right)_{k \geq \kappa_{0}}$ converges to $g(u)$ in $L_{2}(D)$ by Lemma 2.2 (b) and that $\left(u_{k}-k\right)_{+}=0$ for $k \geq \kappa_{0}$. This proves Theorem 1.3.

\subsection{Proof of Corollary 1.5}

Suppose (1.3), (1.8), and (1.9) and let $u \in \bar{S}_{0}$ be any solution to the variational inequality (1.1). Note that (1.8) and (1.9) imply $g(u)=\mathfrak{g}(u)$ in (1.5a). In particular, the function $g(u)$ is non-negative. Assume now for contradiction that the coincidence set $\mathcal{C}(u)$ is not an interval. Then there are $-L<a<b<L$ with $u(a)+H=\partial_{x} u(a)=u(b)+H=\partial_{x} u(b)=0$ and $u>-H$ in $I:=(a, b)$. We may then argue as in the proof of Proposition 2.6 to conclude that $z:=u+H \in H^{4}(I) \cap H_{D}^{2}(I)$ is a strong solution to the boundary value problem

$$
\begin{gathered}
\beta \partial_{x}^{4} z-\tau \partial_{x}^{2} z=-g(u) \text { in } I, \\
z=\partial_{x} z=0 \text { on } \partial I .
\end{gathered}
$$

Another application of a version of Boggio's comparison principle [4, 8, 11, 17] implies $z=u+H \leq 0$ in $I$. But this contradicts $u>-H$ in $I$.

Acknowledgements Open Access funding provided by Projekt DEAL.

\section{Compliance with ethical standards}

Conflict of interest The authors declare that they have no conflict of interest.

Open Access This article is licensed under a Creative Commons Attribution 4.0 International License, which permits use, sharing, adaptation, distribution and reproduction in any medium or format, as long as you give appropriate credit to the original author(s) and the source, provide a link to the Creative Commons licence, and indicate if changes were made. The images or other third party material in this article 
are included in the article's Creative Commons licence, unless indicated otherwise in a credit line to the material. If material is not included in the article's Creative Commons licence and your intended use is not permitted by statutory regulation or exceeds the permitted use, you will need to obtain permission directly from the copyright holder. To view a copy of this licence, visit http://creativecommons.org/licenses/by/4.0/.

\section{References}

1. Amann, H., Escher, J.: Analysis. III. Birkhäuser Verlag, Basel (2009)

2. Ambati, V. R., Asheim, A., van den Berg, J. B., van Gennip, Y., Gerasimov, T., Hlod, A., Planqué, B., van der Schans, M., van der Stelt, S., Vargas Rivera, M., Vondenhoff, E.: Some studies on the deformation of the membrane in an RF MEMS switch. In: Proceedings of the 63rd European Study Group Mathematics with Industry. Bokhove, O., Hurink, J., Meinsma, G., Stolk, C., and Vellekoop, M., eds., CWI Syllabus, Netherlands, 1 2008, Centrum voor Wiskunde en Informatica, pp. 65-84.http://eprin ts.ewi.utwente.nl/14950

3. Bernstein, D. H., Guidotti, P.: Modeling and analysis of hysteresis phenomena in electrostatic zipper actuators. In: Proceedings of Modeling and Simulation of Microsystems, Hilton Head Island, SC, pp. 306-309 (2001)

4. Boggio, T.: Sulle funzioni di Green d'ordine $m$. Rend. Circ. Mat. Palermo 20, 97-135 (1905)

5. Esposito, P., Ghoussoub, N., Guo, Y.: Mathematical analysis of partial differential equations modeling electrostatic MEMS. Courant Lecture Notes in Mathematics, Courant Institute of Mathematical Sciences, vol. 20. New York; American Mathematical Society, Providence, RI (2010)

6. Flores, G., Mercado, G., Pelesko, J. A., Smyth, N.: Analysis of the dynamics and touchdown in a model of electrostatic MEMS. SIAM J. Appl. Math., 67, pp. 434-446 (electronic) (2006/07)

7. Gazzola, F., Grunau, H.-C., Sweers, G.: Polyharmonic boundary value problems. Lecture Notes in Mathematics, vol. 1991. Springer, Berlin (2010)

8. Grunau, H.-C.: Positivity, change of sign and buckling eigenvalues in a one-dimensional fourth order model problem. Adv. Differ. Equ. 7, 177-196 (2002)

9. Guo, Y., Pan, Z., Ward, M. J.: Touchdown and pull-in voltage behavior of a MEMS device with varying dielectric properties. SIAM J. Appl. Math., 66, pp. 309-338 (electronic) (2005)

10. Laurençot, Ph., Nik, K., Walker, Ch.: Energy minimizers for an asymptotic MEMS model with heterogeneous dielectric properties. Preprint, (2020)

11. Laurençot, Ph., Walker, Ch.: Sign-preserving property for some fourth-order elliptic operators in one dimension or in radial symmetry. J. Anal. Math. 127, 69-89 (2015)

12. Laurençot, Ph., Walker, Ch.: Some singular equations modeling MEMS. Bull. Amer. Math. Soc. (N.S.), 54, pp. 437-479 (2017)

13. Laurençot, Ph., Walker, Ch.: Shape derivative of the Dirichlet energy for a transmission problem. To appear in Arch. Rational Mech. Anal. (arXiv:1901.07257)

14. Lindsay, A. E., Lega, J., Glasner, K. G.: Regularized model of post-touchdown configurations in electrostatic MEMS: equilibrium analysis. Phys. D 280-281, 95-108 (2014)

15. Lindsay, A. E., Lega, J., Glasner, K. G.: Regularized model of post-touchdown configurations in electrostatic MEMS: Interface dynamics. IMA J. Appl. Math. 80, 1635-1663 (2015)

16. Nečas, J.: Les méthodes directes en théorie des équations elliptiques, Masson et Cie, Éditeurs. Academia, Prague (1967)

17. Owen, M. P.: Asymptotic first eigenvalue estimates for the biharmonic operator on a rectangle. J. Differ. Equ. 136, 166-190 (1997)

18. Pelesko, J. A.: Mathematical modeling of electrostatic MEMS with tailored dielectric properties. SIAM J. Appl. Math., 62, pp. 888-908 (2001/02)

19. Pelesko, J. A., Bernstein, D. H.: Modeling MEMS and NEMS. Chapman \& Hall/CRC, Boca Raton, FL (2003)

20. Yang, Y., Zhang, R., Zhao, L.: Dynamics of electrostatic microelectromechanical systems actuators. J. Math. Phys., 53, pp. 022703, 13 (2012)

Publisher's Note Springer Nature remains neutral with regard to jurisdictional claims in published maps and institutional affiliations. 